$\mathrm{DE}$

M E D I C I N A

T R O P I C A L

$\mathrm{DE}$

S ÃO PAULO

JOURNAL OF THE SÃO PAULO INSTITUTE OF TROPICAL MEDICINE

1 Universidade Federal do Rio de Janeiro, Faculdade de Medicina, Rio de Janeiro, Rio de Janeiro, Brazil

2Universidade Federal do Rio, Instituto de Puericultura e Pediatria Martagão Gesteira, Rio de Janeiro, Rio de Janeiro, Brazil

Correspondence to: Clemax Couto Sant'Anna

Universidade Federal do Rio de Janeiro, Faculdade de Medicina, R. Bruno Lobo, 50, CEP 21941-901, Rio de Janeiro, RJ, Brazil Tel: +55 2125904891

E-mail: clemax01@gmail.com

Received: 27 July 2020

Accepted: 25 August 2020

\section{Calvarial tuberculosis: two cases in children}

\author{
Clemax Couto Sant'Anna ${ }^{\circledR}$, Pedro Luiz Ferreira Perinei ${ }^{\circledR}$, Ana Carolina \\ Machado de Pessôa ${ }^{\circledR}$, Ekaterini Goudouris ${ }^{\left({ }^{D}\right.}$, Ana Cristina Cisne Frota ${ }^{\left({ }^{\circ}\right.}$
}

\section{ABSTRACT}

Tuberculosis of the skull or calvarial tuberculosis (CTB) is rare. The literature until 2019 reported less than 60 cases of CTB in childhood. The authors describe two patients with CTB associated with other manifestations of TB, such as: spine and rib injuries, peripheral adenopathy, hepatic and splenic involvement who improved with chemotherapy. The patients were a four-year-old and an eight-year-old child, whose diagnoses were confirmed by histopathological, bacteriological or molecular investigation. Both were not infected with the human immunodeficiency virus (HIV) and did not need orthopedic treatment.

KEYWORDS: Tuberculosis. Calvarial tuberculosis. Children. Skeletal tuberculosis. Extrapulmonar tuberculosis.

\section{INTRODUCTION}

Tuberculosis (TB) of the skull or calvarial tuberculosis (CTB) is estimated to occur in only $1 \%$ of osteoarticular TB cases. The disease begins with the infection of the spinal cord, which can spread to both, the inner and outer plates of the skullcap, causing bone destruction and formation of granulation tissue. The infection can be contained by a fibrotic envelope layer formation or it can extend through each border. If the process is rapid, bone sequestration may occur with formation of caseous necrosis ${ }^{1,2}$. The involvement of the external border appears as a palpable nodule, usually painless and soft, with or without fistulization. Lesions of the internal border lead to extra-dural granulation. Although rare, most cases of CTB occur in the first two decades of life, mainly due to the mycobacteria greater affinity for spongy bone. However, infants escape this rule, since there is a low amount of this type of bone in this age group ${ }^{3-5}$.

The first description of CTB in the literature is that of an 18-year-old male adolescent who died in 1842 with multiple bone, lungs, elbows and thighs injuries. There were about 370 cases of CTB in adults and children in the English literature until December 2019,6,7-11. From 2000 to 2019 there were just over 60 occurrences in children under 20 years old in the English and French literature. Acid-fast bacilli (AFB) were found in the bone lesion in about $20 \%$ of these patients and granuloma (compatible or suggestive with TB) was present in most cases ${ }^{2,5,8,9}$. In Brazil, we have not identified any reports of childhood CTB.

The most affected bones of the skull are: parietal, frontal and occipital. Lesions are unique or disseminated, usually lytic, although sclerotic lesions can also occur. According to the literature, in about $10 \%$ of children there was association with brain impairment. Lung was affected in $5 \%$ of these patients ${ }^{2,5,8}$. 
The authors describe two cases of CTB associated with other manifestations of TB in the spine, ribs, along with peripheral adenopathy, hepatic and splenic involvement.

\section{Case report 1}

A 4-year-old boy was admitted with fever for more than 30 days, low back pain, peripheral and abdominal adenomegaly, hepatomegaly and splenomegaly. The abdominal ultrasound showed disseminated nodules in the liver and spleen. The chest X-ray showed a lytic lesion in the $11^{\text {th }}$ right costal arch and a slight upper left pulmonary infiltrate. Tuberculin skin test (TST) with PPD Rt-23 was $>10 \mathrm{~mm}$. He was not vaccinated with BCG. Anti-HIV serology was negative.

A cervical lymph node biopsy showed nonspecific inflammatory findings and granulomatous hepatitis was found in liver biopsy. Computed tomography (CT) and magnetic resonance imaging showed osteolytic lesions with partial destruction of $\mathrm{C} 6$ and $\mathrm{C} 7$ vertebral bodies and a paravertebral abscess.

Afterwards, he presented limited cervical movement, without neurological symptoms. A neck brace was prescribed and treatment for TB with rifampicin, isoniazid and pyrazinamide (RHZ) was started. During his hospitalization, it was found out that the patient had previously remained in a ward in contact with a patient undergoing TB treatment, about six months prior to the onset of his symptoms.

Sixty days after starting treatment, the patient no longer had fever and and hepatic and splenic granulomas were calcified. There was a progressive improvement in bone lesions, but a painless nodular lesion appeared in the skullcap, without neurological symptoms. A new cranial CT scan showed multiple lytic lesions on the external board of the left frontal region (Figure 1), left parietal and right occiput, with soft tissues edema, without subgaleal fat involvement. Another lesion with an extradural soft tissue component was identified in the right frontal region and reached both, the inner and the outer bone layers measuring $1 \mathrm{~cm}$ in diameter. Excisional biopsy of the frontal lesion showed AFB-positive granulomas (Figure 2). Mycobacterial cultures were negative in all analyzed materials. The patient underwent immunological investigation and the only alteration found was the lack of response to IL-12 and also to IFN- $\gamma$ in an in vitro functional assay with mycobacteria and BCG vaccine ${ }^{12}$.

After six months of TB treatment, all bone lesions involuted, with consolidation and fusion of C6 and C7 vertebrae, complete resolution of paravertebral abscess and cranial nodules. He underwent treatment for 12 months.

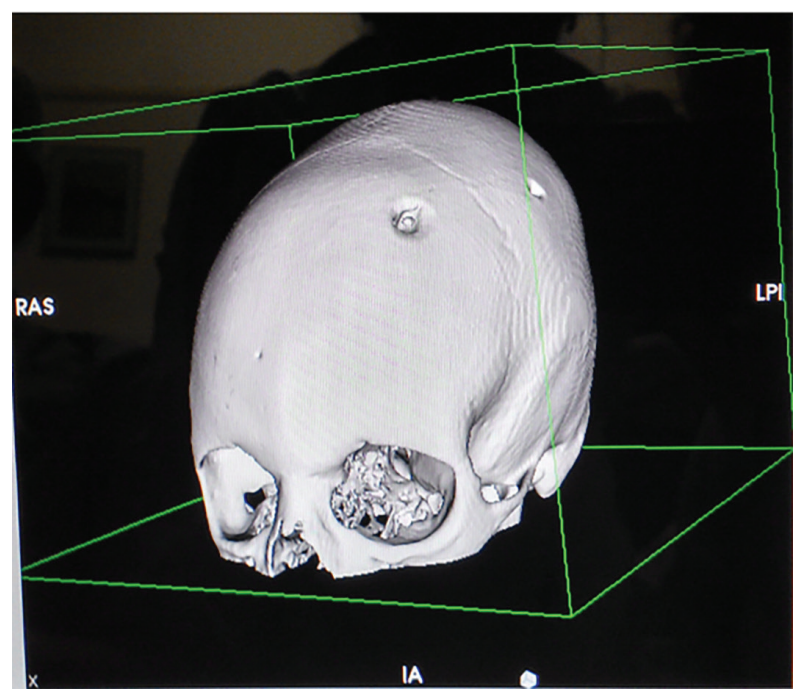

Figure 1 - Cranial CT with reconstruction: multiple lytic lesions on the external board of the left frontal region.

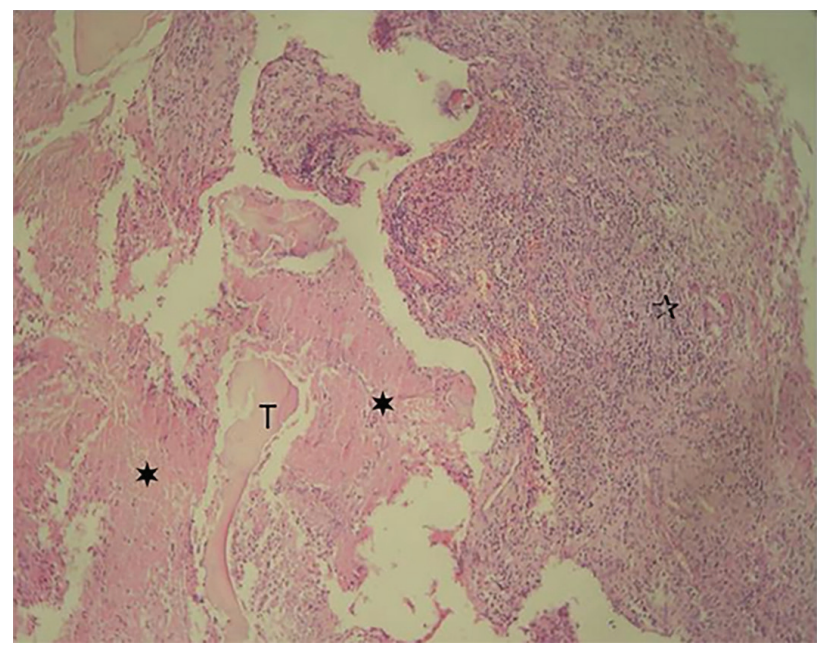

Figure 2 - Areas of necrosis $\left({ }^{*}\right)$ in the center of bone trabeculae (T) involved by chronic inflammatory reaction - HE X 100 .

\section{Case report 2}

A 9-year-old boy had an increase in abdominal volume associated with nonspecific abdominal pain and fever for 2 months, evolving without improvement of symptoms, so he was admitted for investigation presenting a swelling in the left foot. After 3 weeks the patient presented a painless swelling of frontal region, without other signs of inflammation or cervical lymph node enlargement. Painful swelling also appeared in the left ankle. There was no epidemiological history of TB. TST with PPD Rt23 was $11 \mathrm{~mm}$ and the HIV-test was negative. Chest X-ray was normal; chest CT revealed the presence of $1.1 \mathrm{~cm}$ lymph nodes in the pre-tracheal region, $0.8-1.1 \mathrm{~cm}$ near the right anterior costophrenic sinus and other smaller lymph nodes in anterior mediastinum and infracarinal region. In addition, 
there was a small right pleural effusion without lesions in pulmonary parenchyma. Ultrasonography and abdominal $\mathrm{CT}$ performed one week before revealed the presence of a moderate ascite, enlarged liver (regular contour and homogeneous density), small lymph nodes along the right common iliac vessels and $1.9 \times 1.2 \mathrm{~cm}$ nodular image next to the left diaphragmatic dome (probably a lymph node). The cranial CT showed a frontal lytic lesion in the midline measuring $2 \mathrm{~cm}$ and a thickness of $0.7 \mathrm{~cm}$; the internal bone layer was intact and there was a rupture of the external plate extending beyond the extra-cranial layer (Figure 3). The extracranial segment had a soft tissue density of $1.6 \times 0.7 \mathrm{~cm}$, with normal cerebral parenchyma, ventricular system and cisterns. The skull biopsy showed a chronic inflammatory process with granuloma and multinucleated giant cells; Ziehl Neelsen, Groccot and PAS staining did not show microorganisms. A left ankle synovium biopsy was also performed. The Xpert MTB/RIF test on synovium material for Micobacterium tuberculosis complex was positive. The patient underwent an immunological investigation but it was not possible to show an altered functional assay for the IFN-g/IL12 axis, with no response to either cytokine ${ }^{12}$.

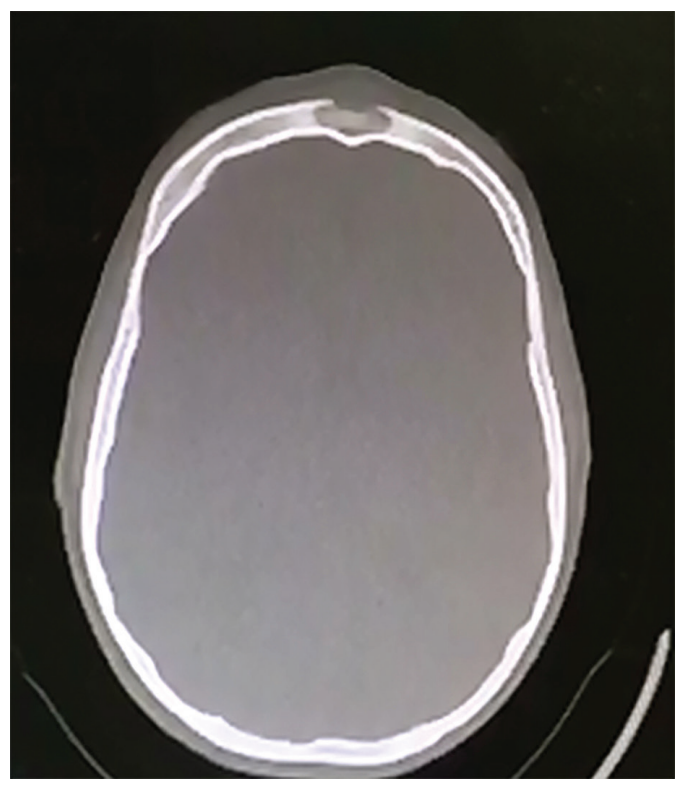

Figure 3 - Cranial CT: frontal lytic lesion in the midline with a diameter of $2 \mathrm{~cm}$ and a thickness of $0.7 \mathrm{~cm}$.

The patient received RHZ regimen to treat TB for 9 months with complete frontal mass regression. He maintained the small joint movement restriction with limited left ankle flexion. He improved his weight significantly and completed treatment after 9 months. The frontal mass disappeared, maintaining the small joint restriction with residual limitation of left ankle flexion. There was an important weight gain.

\section{DISCUSSION}

In general, Mycobacterium tuberculosis is not isolated by cultures in extrapulmonary forms of TB. In one patient, however, the bone smear microscopy was positive and in the other patient the Xpert MTB/RIF was positive in the ankle synovium biopsy. In addition, the histopathological examination showed TB-compatible granuloma in both patients. In the literature, there are reports of only six patients aged 4 years or less, as our patient number 1 and eight reports of children between 5 and 9 years old as our patient number 2. In the last five years, most articles were from India ${ }^{1,2,4,10,11,13}$.

Patient 1 was not vaccinated by BCG. Theoretically, BCG could protect against severe TB forms, as this disseminated presentation could be considered. On the other hand, he would have been infected at a young age, if we consider the in-hospital contact as the mode of acquisition of the infection. A possible genetic defect would be a STAT1 LOF, but, unfortunately, it was not possible until now to carry out to carry out a genetic test.

The second patient, however, had been vaccinated by BCG at birth. Our patient 2 had several manifestations of osteoarticular TB, similar to cases reported in India ${ }^{2,8}$. An important suspicion in this patient would be Mendelian Susceptibility to Mycobacteria Disease (MSMD). However, this patient did not maintain a proper outpatient follow-up and it was not possible to perform the in vitro functional assay with mycobacteria and BCG vaccine to assess his IFN-g / IL-12 axis functionality.

The other manifestations of TB in our patients showed the greater ability of primary TB to involve multiple organs, as was demonstrated by the multiple lesions presented by both children. The involvement of the skull, although rare, should raise suspicion for the possibility of CTB. Other causes such as metastases, histiocytosis, pyogenic, fungal or mycobacterial osteomyelitis must also be evaluated ${ }^{8,9}$.

There is no consensus on the regimen and duration of treatment. Our patients were treated with an RHZ regimen (up to 12 months). According to the literature, surgical treatment was used in association with drugs in most cases in adults published since 2000. Our patients did not need surgical treatment, similarly to most other children in the literature ${ }^{11}$.

\section{ACKNOWLEDGMENTS}

The authors thank to Dr. Jandra Corrêa de Lacerda (IPPMG- UFRJ); CCS was supported by Conselho Nacional de Pesquisa e Desenvolvimento (grant No 302973/2019-2). 


\section{AUTHORS' CONTRIBUTIONS}

CCS, PLP and ACCF had primary responsiblity for study development, the final data analysis and writing the manuscript; PLP, and ACMP were responsible for patient screening and contributed to the writing of the manuscript; CCS, ACCF and EG participated in the enrollment of patients, analytical framework for the study, critical review and contributed to the writing of the manuscript. All the authors agreed with the manuscript submission.

\section{CONFLICT OF INTERESTS}

None were declared.

\section{REFERENCES}

1. Dawar P, Gupta DK, Sharma BS, Jyakumar A, Gamanagatti S. Extensive calvarial tuberculosis presenting as exophytic ulcerated growth on scalp in an infant: an interesting case report with review of literature. Childs Nerv Syst. 2013;29:1215-8.

2. Datta SG, Bhatnagar V, Pan S, Mehta R, Sharma C. Primary calvarial tuberculosis: a report of three cases. Br J Neurosurg. 2019;33:196-201.

3. Mukherjee KK, Kaushik R, Nada R, Khosla VK, Khandelwal N, Kak VK. Calvarial tuberculosis. Surg Neurol. 2002;57:195202.
4. Shah JR. Calvarial tuberculosis features in 3 cases. West Engl Med J. 2013;112:2.

5. Das S, Nayar A, Paul DK, Basu S, Sen S, Bhattacharya M, et al. Calvarial tuberculosis with multiple intracranial tuberculomas: a rare association in a severely malnourished child with disseminated tuberculosis. J Pediatr Infect Dis. 2016;11:42-7.

6. Diyora B, Kumar R, Modgi R, Sharma A. Calvarial tuberculosis: a report of eleven patients. Neurol India. 2009;57:607-12.

7. Pandey AS, Surana A. Tuberculous osteomyelitis of the bone flap following craniotomy. Indian J Tuberc. 2011;58:129-31.

8. Pati S, De S, Ghosh TN, Gosh MK. Multifocal pure tubercular osteomyelitis: an usual presentation in childhood. Indian J Tuberc. 2017;64:136-40

9. Mandal A, Singh A. The many faces of tuberculosis. Int J Pediatr Adolesc Med. 2017;4:112-4

10. Boubrika M, Ait Benalib S. Ostéite tuberculeuse de la voûte du crâne: à propos de deux cas. Arch Pediatr. 2011;18:397-400.

11. Muzundar D, Vedanatm R, Chandrashekhar D. Tuberculosis of the central nervous system in children. Childs Nerv System 2018;34:1925-35.

12. Bustamante J, Picard C, Fieschi C, Filipe-Santos O, Feinberg $\mathrm{J}$, Perronne $\mathrm{C}$, et al. A novel X-linked recessive form of Mendelian susceptibility to mycobaterial disease. J Med Genet. 2007;44:e65.

13. Sridharan S, Balasubramanian D. Primary calvarial tuberculosis. Surg Neurol Int 2017;8:126. 